Authors

Robert K. Shelton, Seth M. Foreman, Long-Sheng Ma, John L. Hall, Henry C. Kapteyn, Margaret M.

Murnane, Mark Notcutt, and Jun Ye 


\title{
Subfemtosecond timing jitter between two independent, actively synchronized, mode-locked lasers
}

\author{
Robert K. Shelton, Seth M. Foreman, Long-Sheng Ma, John L. Hall, Henry C. Kapteyn, Margaret M. Murnane, \\ Mark Notcutt, and Jun Ye* \\ JILA, National Institute of Standards and Technology and University of Colorado, Department of Physics, University of Colorado, \\ Boulder, Colorado 80309-0440
}

Received August 30, 2001

\begin{abstract}
With the implementation of a fast-bandwidth servo, along with improved laser construction and associated better passive stability, we have achieved subfemtosecond relative timing jitter between two independent, actively synchronized, mode-locked Ti:sapphire lasers. Timing jitter of $0.58 \mathrm{fs}$ is obtained with a $160-\mathrm{Hz}$ observation bandwidth over several seconds. Within a 2-MHz observation bandwidth, the timing jitter is $1.75 \mathrm{fs}$. Excellent repeatability and rapid speed in setting an arbitrary time delay between two pulses are also demonstrated. (c) 2002 Optical Society of America

OCIS codes: $320.0320,140.7090$.
\end{abstract}

The merger of ultrafast laser techniques and precision frequency metrology has resulted in recent dramatic progress in a number of fields, including optical frequency measurement, ${ }^{1,2}$ optical clocks, $, 3,4$ and carrier-envelope phase control. ${ }^{5,6}$ We have also demonstrated that combined time and frequency active stabilization can allow one to tightly synchronize two separate, passively mode-locked femtosecond lasers. $^{7}$ The remaining rms timing jitter during an observation time of tens of seconds was shown to be less than $30 \mathrm{fs}$ within a $50-\mathrm{kHz}$ bandwidth and less than $5 \mathrm{fs}$ within a $160-\mathrm{Hz}$ bandwidth. This capability has allowed phase locking between the carrier waves of the two synchronized Ti:sapphire lasers. ${ }^{8}$ However, since the characteristic timing jitter was larger than the optical cycle period, phase locking could occur for only a few tens of microseconds at a time. Coherent optical pulse synthesis from these lasers was subsequently demonstrated. ${ }^{8}$ The ultimate goal of this research would be to demonstrate an arbitrary light waveform generator capable of synchronizing and phase locking arbitrary, separate mode-locked lasers with distinct optical properties. It is thus desirable to enhance the precision level of synchronization such that the remaining timing jitter between different lasers would be less than the oscillation period of the optical carrier wave, namely, $2.7 \mathrm{fs}$ for Ti:sapphire lasers centered around $800 \mathrm{~nm}$. One possible approach uses cross-phase modulation to passively synchronize two mode-locked lasers that share the same intracavity gain medium. ${ }^{9}$ However, the requirement of sharing an intracavity element limits the flexibility and general applicability of this technique.

This Letter presents the latest results of our active synchronization of two passively mode-locked lasers. We have substantially improved the passive stability of both lasers and have implemented a fast servo loop to stabilize the laser repetition frequency. When the synchronization loop is activated, the rms timing jitter between the two Ti:sapphire lasers observed over several seconds is $0.58 \mathrm{fs}$ at a $1-\mathrm{ms}$ averaging time ( $160-\mathrm{Hz}$ bandwidth). The timing jitter increases to only $1.75 \mathrm{fs}$ if the observation bandwidth is ex- tended beyond $1 \mathrm{MHz}$. Since the timing jitter is below the period of an optical cycle, we now have a system that could maintain a carrier phase lock on much longer time scales than in our previous work. ${ }^{7,8}$ All-electronic control allows any arbitrary time delay between two pulse trains to be set with excellent repeatability and at a short settling time of $\sim 60 \mu \mathrm{s}$. The limiting factor in achieving the lowest timing-jitter noise is the intrinsic noise floor of the phase detector used in the stabilization loop.

The two lasers are both located on a temperature-controlled, 5-cm-thick, solid aluminum baseplate, which is decoupled from the vibration noise of the table by a set of supporting feet made from rubber. In addition, asymmetrically positioned lead plates are tightly bonded to the bottom side of the baseplate to damp the vibration modes. The vibration noise measured on top of the baseplate is generally reduced by $10 \mathrm{~dB}$ or more within the frequency range of $100 \mathrm{~Hz}$ to $6 \mathrm{kHz}$ compared with the noise on the optical table. We employ two low-threshold Kerr-lens mode-locked Ti:sapphire lasers that each produce a mode-locked average power of more than $300 \mathrm{~mW}$ with 2.5 -W pump power at $532 \mathrm{~nm} .{ }^{10}$ The beam height inside the laser cavities is only $6 \mathrm{~cm}$ above the baseplate, which should enhance stability. We find that to achieve the lowest possible timing jitter it is critical to enclose both lasers and their pump beams to shield them from blowing dust and convection currents.

To synchronize the two lasers (A and $\mathrm{B}$ ), we use two phase-locked loops (PLLs) working at different timing resolutions. For these experiments, laser A remains free running. Both PLLs operate solely on laser B. A similar diagram of our feedback system may be found in our previous work. ${ }^{7}$ One PLL compares and locks the fundamental repetition frequencies $(100 \mathrm{MHz})$ of the two lasers. The phase shift between the two $100-\mathrm{MHz}$ signals can be used to control the (coarse) timing offset between the two pulse trains with a full dynamic range of $5 \mathrm{~ns}$. The second, high-resolution, PLL compares the phase of the 140th harmonic of the two repetition frequencies, i.e., at $14 \mathrm{GHz}$. This second loop provides enhanced phase stability of the repetition frequency when it 
supplements and then replaces the first PLL. A transition of control from the first to the second PLL can cause a jump in the timing offset by at most $35.71 \mathrm{ps}$ (one half of one $14-\mathrm{GHz}$ cycle), whereas the adjustable range of the $14-\mathrm{GHz}$ phase shifter is $167 \mathrm{ps}$. The servo action on laser $\mathrm{B}$ is carried out by a combination of transducers, including a fast piezoactuated small mirror $(4 \mathrm{~mm}$ in diameter and $2.5 \mathrm{~mm}$ thick), a regular mirror mounted on a slow piezo with a large dynamic range $(2.7 \mu \mathrm{m})$, and an acoustooptic modulator placed in laser B's pump beam to help with the fast noise. The unity gain frequency of the servo loop is $\sim 200 \mathrm{kHz}$, and the loop employs three integrator stages in the low-frequency region.

To characterize the timing jitter, we focus the two pulse trains so that they cross in a thin BBO crystal cut for sum frequency generation (type I SFG). The crossed-beam geometry produces a non-fringe-resolved SFG cross-correlation signal. The Gaussian cross-correlation peak (obtained when the two lasers are free running) is $\sim 161 \mathrm{fs}$ FWHM. (No extracavity dispersion compensation is used, so the would-be 20 -fs laser pulses are broadened.) The time axis of the cross correlation is calibrated by measurement of the difference between the two laser repetition rates when the cross correlation is recorded. The calibrated slope of the cross-correlation signal near the middle height can be used to determine the relative timing jitter between the two lasers from the corresponding intensity fluctuations. We record the intensity fluctuations of the SFG signal over a period of several seconds, using two different low-pass bandwidths, $160 \mathrm{~Hz}$ and $2 \mathrm{MHz}$, which suppress the pulsed nature $(100 \mathrm{MHz})$ of the SFG signal amplitude and permit the study of intensity noise on a cw basis.

The top trace in Fig. 1(a) shows that the SFG signal, recorded with a $2-\mathrm{MHz}$ bandwidth, shows only digitalization noise when the two laser pulses overlap maximally (at the top of the cross-correlation peak). In this case, the SFG signal is least sensitive to the timing jitter and its intensity noise is limited by each individual laser's amplitude fluctuation. The two middle traces are recorded with $2-\mathrm{MHz}$ and $160-\mathrm{Hz}$ bandwidths when the timing offset between the two pulse trains is adjusted to yield the half-intensity level of the SFG signal. Timing jitter is calculated from the intensity noise by use of the slope of the correlation peak, with the scale of the jitter indicated on the vertical axis of Fig. 1(a). The rms timing noises are thus determined to be $1.75 \mathrm{fs}$ at a $2-\mathrm{MHz}$ bandwidth and $0.58 \mathrm{fs}$ at a $160-\mathrm{Hz}$ bandwidth. For detection bandwidths above $2 \mathrm{MHz}$, the observed jitter does not increase. We have recorded such stable performance over several seconds. The synchronization lock can be maintained for durations of several hours. However, the intensity stability of the SFG signal is found to correlate strongly with the temperature variations in the microwave cables.

To understand noise contributions at various time scales, we study the SFG signal with a fast Fourier-transform frequency spectrum analyzer. Figures 1(b) and 1(c) display the power spectral density (resolution bandwidth normalized) of the timing noise up to Fourier frequencies of $100 \mathrm{~Hz}$ and $100 \mathrm{kHz}$, respectively. The spikes near dc are artifacts from the analyzer itself. When the spectral noise from Fig. 1(b) is integrated from $1 \mathrm{~Hz}$ to $100 \mathrm{~Hz}$ (avoiding the near-dc artifacts), we calculate a timing jitter of $\sim 0.6 \mathrm{fs}$. When the data set in Fig. 1(c) is integrated from $1 \mathrm{~Hz}$ to $100 \mathrm{kHz}$, the resultant rms timing jitter is $1.4 \mathrm{fs}$. These results are consistent with the direct time-domain data shown in Fig. 1(a).

Since the SFG signal represents a measure from outside the servo loop, the intensity analysis of this signal reflects the true evaluation of the relative timing jitter between the two synchronized lasers. A careful study of the servo error signal inside the feedback loop reveals that a major part of the limitation to the current attainable level of performance is actually due to the intrinsic noise of the $14-\mathrm{GHz}$ phase detector, a double-balanced mixer. Figure 2 displays the power spectral density of the error signal under tight lock, along with that of the mixer noise. Among the five mixers we tested, the best one has an intrinsic phase-noise power spectral density of $2 \times 10^{-13} \mathrm{rad}^{2} / \mathrm{Hz}$ at $14 \mathrm{GHz}$. This phase-noise floor corresponds to a timing noise of $2.5 \times 10^{-5} \mathrm{fs}^{2} / \mathrm{Hz}$. Figure 2(a) shows that the synchronization loop error signal and the mixer noise floor basically overlap across the entire $100-\mathrm{kHz}$ Fourier frequency range. At low frequencies, the servo loop gain is actually slightly too high, such that the noise level of the servo error becomes less than the mixer noise [Fig. 2(b)]. Integration of this (resolution-bandwidth-normalized) intrinsic noise level produces the lowest rms timing jitter limit for the synchronization loop. For example, from Fig. 2(b) we determine the rms timing jitter limit for the $1-\mathrm{Hz}-160-\mathrm{Hz}$ frequency range to be $\left(2.5 \times 10^{-3} \mathrm{fs}^{2} / \mathrm{Hz} \times 160 \mathrm{~Hz}\right)^{1 / 2} \approx 0.63 \mathrm{fs}$.
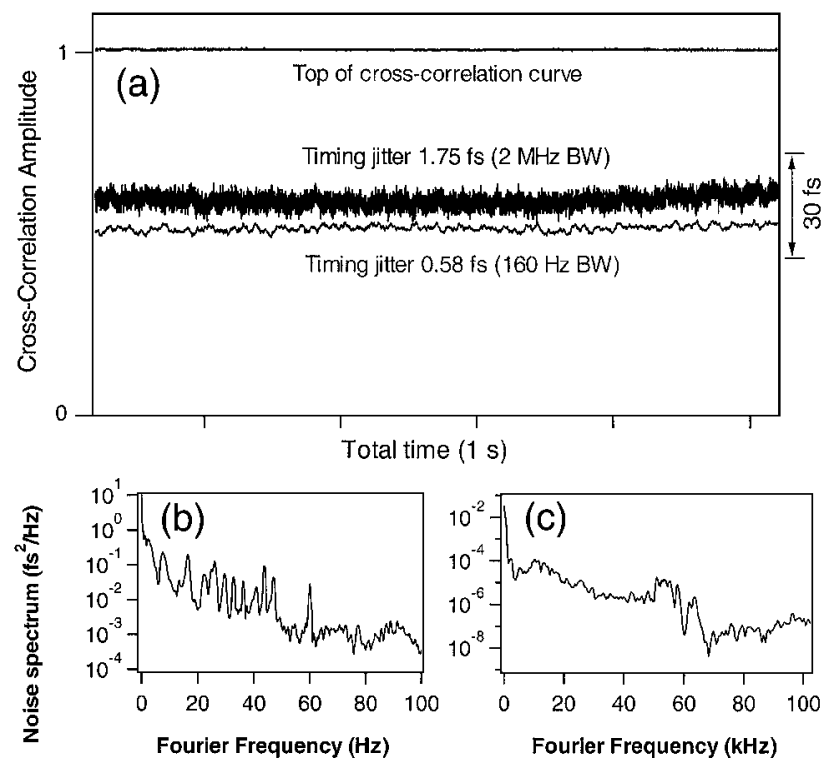

Fig. 1. Timing jitter between two synchronized femtosecond lasers. (a) Time record of noise determined from fluctuations of the SFG intensity. (b) SFG intensity-noise power spectral density from dc to $100 \mathrm{~Hz}$. (c) SFG intensity-noise power spectral density from dc to $100 \mathrm{kHz}$. 

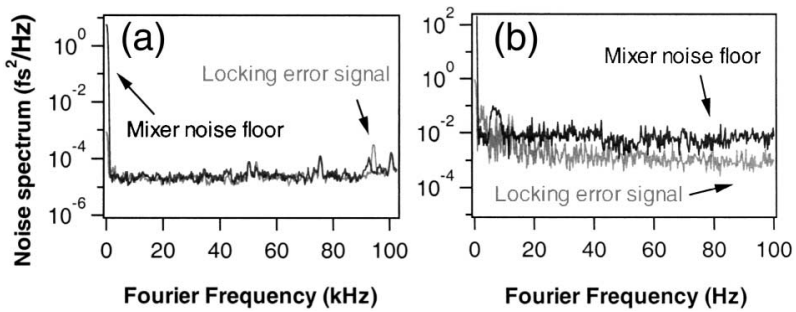

Fig. 2. Power spectral density of the servo error signal and the intrinsic noise of the mixer from dc to $100 \mathrm{kHz}$ (a) and from dc to $100 \mathrm{~Hz}$ (b). These results show that the present performance is limited by the intrinsic noise floor of the best mixer that we have at $14 \mathrm{GHz}$.
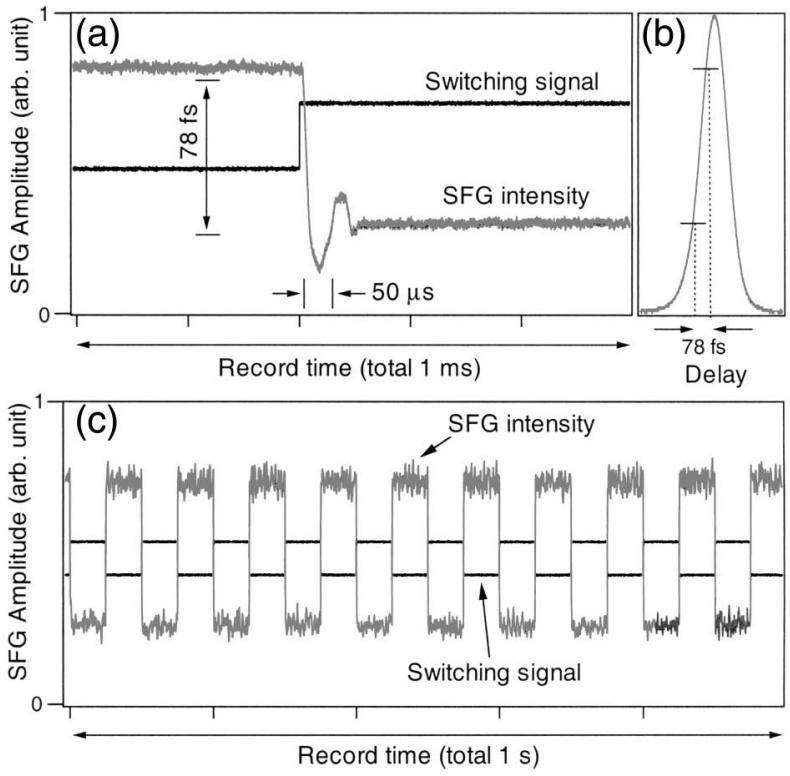

Fig. 3. Controlled switching of time delays between the two pulse trains. (a) Switching dynamics showing a 6.3- $\mu \mathrm{s}$ delay, a $10-\mu \mathrm{s}$ servo attack time, and $\sim 60-\mu \mathrm{s}$ settling time in the real signal after the control signal in the feedback loop is applied. (b) Cross-correlation signal between the two pulse trains. (c) $10-\mathrm{Hz}$ switching sequences recorded in a 1-s period.

For practical ultrafast applications, it is important to show that this low level of timing noise can be obtained at any predetermined timing offset between two laser pulse trains. It is also desirable to realize a reliable setting of the timing offset at high speed and with excellent repeatability. We show a vast improvement from our previous work in terms of precision and settling time. ${ }^{7}$ Figure 3 demonstrates such capabilities of our synchronization system. Figure 3(a) shows fast switching of the pulse delay time by $\sim 78 \mathrm{fs}$, indicated by a jump in the SFG signal. The switching signal applied to the synchronization PLL is also shown. Associated with servo bandwidth limitations, there is a $6.3-\mu$ s delay between the command signal applied to the servo loop and the actual jump in the pulse timing offset. The servo attack time is $\sim 10 \mu \mathrm{s}$ and the pulse timing is settled in $\sim 60 \mu \mathrm{s}$. The precision level of the switched pulse timing is $\sim 1 \mathrm{fs}$. For clarity, we also show the magnitude of the cross-correlation signal in Fig. 3(b). Figure 3(c) displays a 1-s record of continuous switching at a frequency of $10 \mathrm{~Hz}$ between two predetermined pulse delay times. Again, repeatability in timing offset $\sim 1 \mathrm{fs}$. is demonstrated. Compared with a mechanical scanning system, this electronic tuning method thus offers vastly superior performance in terms of repeatability, reliability, and speed, with no noticeable hysteresis.

The all-electronic control of the timing offset between separate lasers is flexible and generally applicable. Combined with the ultralow level of the timing noise, the techniques that we present here should have a wide range of applications. Further reduction of the timing noise can come from detection of even higher harmonics of the repetition frequency. However, we will quickly run out of the practical range of microwave technology. An attractive alternative would be to use optical means. One can use either a single highly stable $\mathrm{cw}$ laser ${ }^{2,11}$ or a common stable optical resonator ${ }^{12}$ to control a high-order harmonic of the repetition frequency, well into the terahertz or tens or hundreds of terahertz frequency range. We anticipate that timing noise below $0.1 \mathrm{fs}$ should be achievable.

We thank J. L. Peng for making substantial contributions to the work reported here. This research was funded by the National Science Foundation, NASA, the National Institute of Standards and Technology (NIST), and the Research Corporation. J. L. Hall and J. Ye are Staff Members of the Quantum Physics Division of NIST Boulder.

*Corresponding author; ye@jila.colorado.edu.

\section{References}

1. Th. Udem, J. Reichert, R. Holzwarth, and T. W. Hänsch, Phys. Rev. Lett. 82, 3568 (1999).

2. S. A. Diddams, D. J. Jones, J. Ye, S. T. Cundiff, J. L. Hall, J. K. Ranka, R. S. Windeler, R. Holzwarth, Th. Udem, and T. W. Hänsch, Phys. Rev. Lett. 84, 5102 (2000).

3. S. A. Diddams, Th. Udem, J. C. Bergquist, E. A. Curtis, R. E. Drullinger, L. Hollberg, W. M. Itano, W. D. Lee, C. W. Oates, K. R. Vogel, and D. J. Wineland, Science 293, 826 (2001).

4. J. Ye, L.-S. Ma, and J. L. Hall, Phys. Rev. Lett. 87, 270801 (2001).

5. D. J. Jones, S. A. Diddams, J. K. Ranka, A. Stentz, R. S. Windeler, J. L. Hall, and S. T. Cundiff, Science 288, 635 (2000).

6. A. Apolonski, A. Poppe, G. Tempea, C. Spielmann, Th. Udem, R. Holzwarth, T. W. Hänsch, and F. Krausz, Phys. Rev. Lett. 85, 740 (2000).

7. L.-S. Ma, R. K. Shelton, H. C. Kapteyn, M. M. Murnane, and J. Ye, Phys. Rev. A 64, 021802(R) (2001).

8. R. K. Shelton, L.-S. Ma, H. C. Kapteyn, M. M. Murnane, J. L. Hall, and J. Ye, Science 293, 1286 (2001).

9. A. Leitenstorfer, C. Fürst, and A. Laubereau, Opt. Lett. 20, 916 (1995).

10. K. Read, F. Blonigen, N. Riccelli, M. Murnane, and H. Kapteyn, Opt. Lett. 21, 489 (1996).

11. J. Ye, J. L. Hall, and S. A. Diddams, Opt. Lett. 25, 1675 (2000).

12. R. J. Jones and J.-C. Diels, Phys. Rev. Lett. 86, 3288 (2001). 